\title{
LIMITS OF APPROXIMATE SOLUTIONS OF A TORSION PROBLEM*
}

\author{
BY D. L. HOLL AND E. W. ANDERSON
}

1. Introduction. In the application of Ritz's $\dagger$ method to the solution of a boundary value problem by the direct process of the calculus of variations, one has the difficulty of not knowing what degree of approximation it provides in a given case, except to say that it gives an upper limit to the solution. Analogous to the Ritz method in which the form of the solution (containing parameters determinable by a minimizing process) is approximated by a complete set of independent functions satisfying the boundary conditions but not the differential equation, Trefftz $\ddagger$ has presented a method adaptable to the Dirichlet problem, and this provides a lower bound. In this process the approximating functions are chosen to satisfy only the differential equation. By application of both of these approximating methods upper and lower bounds of the true minimum are provided.

In this paper these methods are applied to the torsion problem of a right prism whose cross section is symmetrical and is bounded by arcs of two confocal parabolas.

2. The Torsion Problem. The torsion problem is the problem $\S$ of determining a function $\phi(x, y)$ such that on the boundary $\Gamma$ of the region $R$, it attains the value

$$
\phi=\frac{\tau}{2}\left(x^{2}+y^{2}\right)+\text { a constant }
$$

and

$$
\nabla^{2} \phi=\phi_{x x}+\phi_{y y}=0
$$

* Presented to the Society, December 30, 1929. Abstract No. 36-3-145.

$\dagger$ W. Ritz, Journal für Mathematik, vol. 135 (1909), pp. 1-61; Collected Works, 1911, pp. 192-250.

$\ddagger$ E. Trefftz, Ein Gegenstück zum Ritzschen Verfahren, Proceedings of the 2d International Congress for Applied Mechanics, Zurich, 1926, p. 131.

$\S$ A. E. H. Love, The Mathematical Theory of Elasticity, 4th edition, 1927, p. 312; Handbuch der Physik, vol. 6, Springer, 1928, p. 143. 
holds in $R$. An equivalent formulation is

$$
\begin{gathered}
\psi=\phi-\frac{\tau}{2}\left(x^{2}+y^{2}\right)+(\text { a constant })=0, \\
\nabla^{2} \psi+2 \tau=0 .
\end{gathered}
$$

The elastic energy per unit length and the twisting couple are, respectively,

$$
\begin{aligned}
& U=D[\psi]=\frac{\mu}{2} \iint\left(\psi_{x}^{2}+\psi_{y}^{2}\right) d x d y, \\
& Q=2 \mu \iint \psi d x d y .
\end{aligned}
$$

For the region $R$, it is convenient to introduce orthogonal curvilinear coordinates by the transformation

$$
2(x+i y)=2 Z=W^{2}=(\alpha+i \beta)^{2},
$$

where constant values of $\alpha$ and $\beta$ are the boundaries of $R$. Equations (2) and (5) remain invariant, and the right member of (1) becomes $(\tau / 8)\left(\alpha^{2}+\beta^{2}\right)^{2}+$ a constant.

3. The Ritz Method. For the approximate solution, one may assume

$$
\psi_{n}(\alpha, \beta)=\sum_{1}^{n} c_{i} \xi_{i}(\alpha, \beta)
$$

such that each $\xi_{i}$ vanishes on the boundary, and the $c_{i}$ are so determined that

(9) $I[\psi]=\frac{\mu}{2} \iint\left[\psi_{\alpha}^{2}+\psi_{\beta}^{2}-4 \tau \frac{\psi}{h^{2}}\right] d \alpha d \beta,\left(h=\left|\frac{d W}{d Z}\right|\right)$,

is a minimum. The integral is evaluated over the transformed region $R^{\prime}$. Transforming (4) by (7) yields the Euler-Lagrange condition of (9). $I[\psi]$ may also be considered as the equivalent of minimizing $D[\psi]$ subject to a constant torque $Q$.

It is known that $\psi(\alpha, \beta)$ is such a function that $\xi_{i}$ may be chosen as a complete set of polynomials which converge uniformly to $\psi$, such that 


$$
\lim _{n \rightarrow \infty} \psi_{n}=\psi ; \lim _{n \rightarrow \infty} I\left[\psi_{n}\right]=I[\psi] ; I\left[\psi_{n}\right] \geqq I[\psi] .
$$

The convergence of this process has been shown by Trefftz.*

Since $R$ is symmetric, with boundaries $\alpha^{2}=\beta^{2}=\kappa^{2}$, take

$$
\psi_{n}=\left(\alpha^{4}-\kappa^{4}\right)\left(\beta^{4}-\kappa^{4}\right) \sum_{0}^{n} c_{i} \alpha^{2 i} \beta^{2 i}, \quad(i=0,1,2,3, \cdots) .
$$

Then the equation yields, as a first approximation,

$$
\begin{gathered}
\frac{\partial I\left[\psi_{0}\right]}{\partial c_{0}}=0 \\
c_{0}=\frac{3 \tau}{16 \kappa^{4}}, \quad I_{\min }\left[\psi_{0}\right]=\frac{-4 \mu \tau^{2} \kappa^{8}}{35}, \quad Q_{0}=\frac{8}{35} \mu \tau \kappa^{8} .
\end{gathered}
$$

Using $i=0$ and 1 in (11), the second approximation yields

$$
\begin{gathered}
c_{0}=\frac{209 \tau}{1184 \kappa^{4}}, \quad c_{1}=\frac{143 \tau}{1184 \kappa^{8}}, \\
I_{\min }\left[\psi_{1}\right]=-0.11578 \mu \tau^{2} \kappa^{8} ; Q_{1}=0.23155 \mu \tau \kappa^{8} .
\end{gathered}
$$

4. The Trefftz Method. For the problem defined by (1) and (2) one may assume approximations of the form

$$
\phi_{n}=\lambda_{0}+\sum_{i=1}^{n} \lambda_{i} p_{i}(x, y),
$$

such that $p_{i}$ satisfy (2) and the $\lambda$ factors are determined by

$$
\begin{aligned}
& \int\left(\phi_{n}-\bar{\phi}\right) d s=0, \\
& \int\left(\phi_{n}-\bar{\phi}\right) \frac{\partial p_{i}}{\partial n} d s=0,(i=1,2,3, \cdots, n) .
\end{aligned}
$$

The normal derivative is along the outward normal and $\bar{\phi}$ is the initial condition (1). Since $\phi$ is a regular potential function, $p_{i}$ may be chosen as a complete set of plane harmonic polynomials $\dagger$ such that

* E. Trefftz, Konvergenz und Fehlerschätzung beim Ritzschen Verfahren, Mathematische Annalen, vol. 100 (1928), p. 503.

$\dagger$ E. Trefftz, loc. cit. 


$$
\lim _{n \rightarrow \infty} \phi_{n}=\phi, \lim _{n \rightarrow \infty} D\left[\phi_{n}\right]=D[\phi], D\left[\phi_{n}\right] \leqq D[\phi] .
$$

The defining relation (3) shows that

$$
D[\phi]=I[\psi]+\mu \tau^{2} I_{0} / 2,
$$

where the last term is a constant and $I_{0}$ is the moment of inertia of the section. Hence by this method

$$
I\left[\psi_{n}\right] \leqq I[\psi] .
$$

As a first approximation (14) may be chosen of the form

$$
\phi_{1}=\lambda_{0}+\lambda_{1}\left(\alpha^{4}-6 \alpha^{2} \beta^{2}+\beta^{4}\right) ;
$$

then (15), (9) and (6) yield respectively

$$
\begin{aligned}
\lambda_{0} & =\frac{8}{45} \tau \kappa^{4}, \lambda_{1}=-\frac{5 \tau}{72}, \\
I_{\max }\left[\psi_{1}\right] & =\frac{-16}{135} \mu \tau^{2} \kappa^{8}, \quad Q_{1}=\frac{32}{135} \mu \tau \kappa^{8} .
\end{aligned}
$$

Adding $\lambda_{2}\left(\alpha^{8}-28 \alpha^{6} \beta^{2}+70 \alpha^{4} \beta^{4}-28 \alpha^{2} \beta^{6}+\beta^{8}\right)$ to (17) gives the second approximation,

$$
\begin{gathered}
\lambda_{0}=0.17856 \tau \kappa^{4}, \quad \lambda_{1}=-0.05829 \tau, \lambda_{2}=0.00501 \tau / \kappa^{4} ; \\
I_{\max }\left[\psi_{2}\right]=-0.11690 \mu \tau^{2} \kappa^{8}, \quad \phi_{2}=0.23380 \mu \tau \kappa^{8} .
\end{gathered}
$$

5. Results. The true solution* gives the following results

$$
I[\psi]=-0.11589 \mu \tau^{2} \kappa^{8}, \quad Q=0.23179 \mu \tau \kappa^{8} .
$$

From (12), (13), (18), and (19), the approximate results are

$$
\begin{array}{ll}
I\left[\psi_{0}\right]=-0.11428 \mu \tau^{2} \kappa^{8}, & Q_{0}=0.22857 \mu \tau \kappa^{8} ; \\
I\left[\psi_{1}\right]=-0.11578 \mu \tau^{2} \kappa^{8}, & Q_{1}=0.23155 \mu \tau \kappa^{8} ; \\
I\left[\psi_{1}\right]=-0.11854 \mu \tau^{2} \kappa^{8}, & Q_{1}=0.23707 \mu \tau \kappa^{8} ; \\
I\left[\psi_{2}\right]=-0.11690 \mu \tau^{2} \kappa^{8}, & Q_{2}=0.23380 \mu \tau \kappa^{8} .
\end{array}
$$

From the results it is evident that these methods provide upper and lower bounds to the solution.

* Aıderson and Holl, $A$ torsion problem in curvilinear coordinates, Iowa State College Journal of Science, vol. 3 (1929), No. 3, p. 231. 
6. Recent Work.* K. Friedrichs $\dagger$ has shown how to adjoin a maximum problem to a given minimum problem such that its maximum value equals the minimum value of the original problem. It effects the same purpose as the method of Trefftz (furnishing a lower bound) and has wider applications. Associated with the minimizing of $D[\phi]$ subject to the condition (1), is the problem of maximizing

$$
-\frac{\mu}{2} \iint\left(V_{x}^{2}+V_{y}^{2}\right) d x d y+\mu \int \frac{\partial V}{\partial s} \bar{\phi} d s,
$$

where $V$ satisfies (2). This coincides with the Trefftz process. Results equivalent to (18) were obtained for this torsion problem.

Dr. N. M. Bashu $\ddagger$ has employed the Friedrich principle and set up a maximum problem equivalent to $I[\psi]$ subject to $\psi=0$ on the boundary, and applied it to the deflection of a square membrane and to the torsion of a square prism.

Iowa State College

* Added to the paper since presented to the Society.

$\dagger$ K. Friedrichs, Ein Verfahren der Variationsrechnung, . . , Nachrichten der Gesellschaft der Wissenschaften zu Göttingen, 1929, pp. 13-20; CourantHilbert, Methoden der Mathematischen Physik, vol. I, 2d edition, 1931, p. 199.

$\ddagger$ N. M. Bashu, Philosophical Magazine, (7), vol. 10, No. 66, Nov., 1930, p. 886 . 